\title{
TRES ESPECIES NUEVAS DE MYRTACEAE DE LA SIERRA MADRE ORIENTAL, MÉXICO
}

\author{
Erandi SÁncheZ-Chávez y Sergio Zamudio ${ }^{1}$ \\ Instituto de Ecología, A.C. \\ Centro Regional del Bajío \\ Apartado Postal 386 \\ 61600 Pátzcuaro, Michoacán, México \\ ${ }^{1}$ Autor para la correspondencia: sergio.zamudio@inecol.mx
}

\section{RESUMEN}

Se describen e ilustran tres especies nuevas de Myrtaceae de los estados de Querétaro e Hidalgo: Calyptranthes moctezumae, Eugenia queretaroana y E. huasteca. Calyptranthes moctezumae está relacionada con C. schlechtendaliana y C. schiedeana, de las que se distingue por el menor número de nervaduras por hoja, peciolos de 1 a $5 \mathrm{~mm}$ de largo, y por habitar en bosque tropical caducifolio, subcaducifolio y matorral submontano. Eugenia queretaroana es similar a E. crenularis y E. pueblana, de las que se diferencia por el indumento cobrizo, peciolo terete, hipantio infundibuliforme y frutos elipsoidales con un falso estípite en la base. Por su parte Eugenia huasteca se puede relacionar morfológicamente con E. capuli; sin embargo, la primera presenta indumento simple, pardo rojizo a blanquecino y flores sésiles, características que la separan claramente de cualquier otra especie de este género.

Palabras clave: Calyptranthes, Eugenia, Hidalgo, México, Querétaro.

\begin{abstract}
Three new species of Myrtaceae from Querétaro and Hidalgo, México are described and illustrated: Calyptranthes moctezumae, Eugenia queretaroana and E. huasteca. Calyptranthes moctezumae is related with $C$. schlechtendaliana and C. schiedeana, from which it is distinguished by the lesser number of nerves per leaf, petioles 1 to $5 \mathrm{~mm}$ long, and because it occurs in tropical deciduous and subdeciduous forest, as well as submontane
\end{abstract}


scrub. Eugenia queretaroana is similar to E. crenularis and E. pueblana; nevertheless, it is distinguished by the coppery indumentum, terete petiole, infundibuliform hypanthium, and ellipsoid fruit with a false stipe at base. Eugenia huasteca is related with E. capuli; however, the new species is distinguishable from all the other species in the genus by the brownishred to whitish indumentum of simple hairs and the sessile flowers.

Key words: Calyptranthes, Eugenia, Hidalgo, México, Querétaro.

La familia Myrtaceae se distribuye principalmente en las regiones tropicales del mundo, incluyendo parte de Australia y Nueva Zelanda; está formada por 140 géneros y cerca de 7000 especies (Landrum et al., 2009). En México se registran 10 géneros y aproximadamente 109 especies, mayormente habitantes de las regiones de clima cálido. Calyptranthes tiene alrededor de 100 especies registradas desde México y el Caribe hasta el norte de Argentina; pocas se extienden ampliamente, pues la mayoría se concentran en áreas bien definidas, por lo que McVaugh (1963) reconoció varios centros de endemismo para el género. Por el contrario, Eugenia es uno de los géneros más diversos, con aproximadamente 1000 especies en América, distribuidas desde Florida y el Caribe hasta Argentina, con cerca de 109 adicionales conocidas de África y el sureste de Asia (Mazine et al., 2014).

Durante la revisión de la familia Myrtaceae para el tratamiento de la Flora del Bajío y de Regiones Adyacentes se registraron cuatro géneros (Calyptranthes, Eugenia, Myrcianthes y Psidium) y 14 especies, incluyendo ejemplares de tres taxa de Calyptranthes y Eugenia que no se pudieron asignar a ninguno de los ya conocidos. Después de una serie de exploraciones botánicas en el estado de Querétaro para conocer las plantas en campo y obtener más material, se comprobó que se trata de tres especies nuevas para la ciencia, las que se describen formalmente a continuación.

Calyptranthes moctezumae E. Sánchez-Chávez \& Zamudio sp. nov. Fig. 1.

Tipo: MÉXICO. Querétaro, municipio de Arroyo Seco, Las Adjuntas, $10 \mathrm{~km}$ al SE de Concá, 12 abril 1968, J. Rzedowski 25614 (holotipo: IEB; isotipos: ENCB, IBUG).

Frutex 1-4 m altus; cortex griseo-lutescens in laminas tenues exfolians; ramuli teretes vel leviter compressi bicostati glabri sed trichomatibus aliquis in alabastris praesentibus. Petiolus canaliculatus 1-5 mm longus, lamina foliaris discolora ellip- 


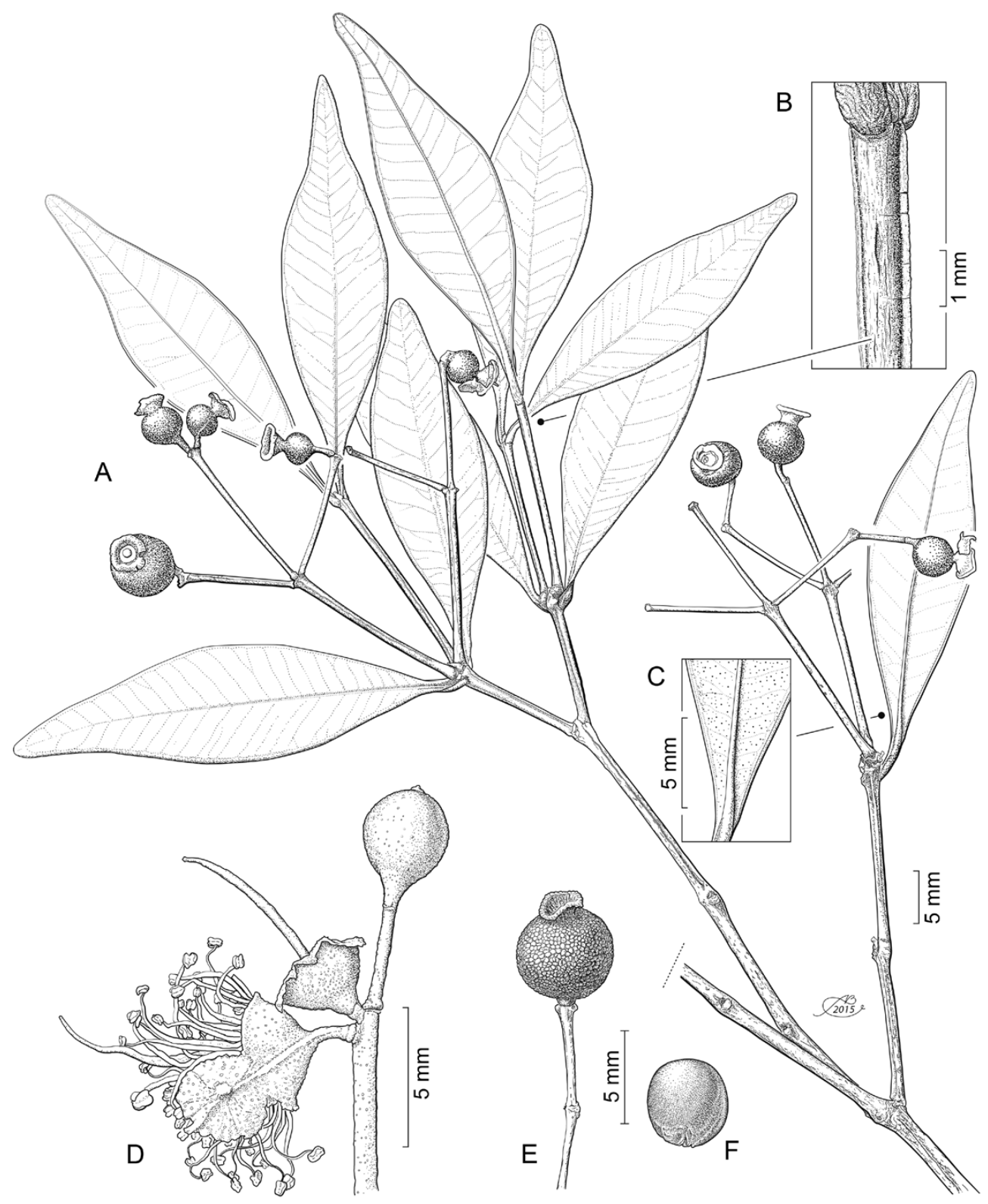

Fig. 1. Calyptranthes moctezumae E. Sánchez-Chávez \& Zamudio. A. rama con frutos; B. acercamiento de la ramilla mostrando el tallo con dos costillas angostas; C. acercamiento del envés de la hoja mostrando el borde revoluto y las glándulas punctiformes; D. segmento de la inflorescencia mostrando la flor caliptrada; E. fruto; F. semilla. Ilustración de Alfonso Barbosa. 
tica ad subrhombica 2.3-5.3 cm longa, 1-2.2 cm lata, coriacea, utrinque glandulis punctiformibus dispersis, nerviis lateralibus $10-15$ in quoque latere inconspicuis ascendentibus angulo $40-50^{\circ}$. Inflorescentiae axillares in dicasiis compositis, pedunculi compressi 1.8-2.5 cm longi. Flores 9-20 per inflorescentiam.

Arbusto de 1 a $4 \mathrm{~m}$ de altura; corteza gris-amarillenta, lisa, exfoliante en láminas delgadas; ramillas teretes a ligeramente comprimidas y 2-costilladas, glabras excepto por algunos tricomas rojizos en las yemas. Peciolo acanalado, de 1 a $5 \mathrm{~mm}$ de largo, por $\pm 1 \mathrm{~mm}$ de ancho, glabro; lámina foliar discolora, verde oscuro, algo lustrosa en el haz, verde pálido en el envés, elíptica a subrómbica, de 2.3 a 5.3 $\mathrm{cm}$ de largo, por 1 a $2.2 \mathrm{~cm}$ de ancho, ápice acuminado a largamente acuminado, el acumen redondeado en la punta, base cuneada, decurrente, margen ligeramente revoluto, vena media plana, ligeramente cóncava hacia la base en el haz, convexa en el envés, nervios laterales inconspicuos, 10 a 15 por lado, generalmente elevados en el haz y envés, ascendentes con un ángulo de inclinación de 40 a 50 grados, nervio marginal levemente arqueado entre los laterales, distante 0.2 a $0.5 \mathrm{~mm}$ del margen, coriácea, con glándulas punteadas dispersas en el haz, pelúcido-punteadas en el envés. Inflorescencias axilares en dicasios compuestos, cerca del ápice de las ramas, de 2 a $5 \mathrm{~cm}$ de largo; pedúnculos comprimidos, amarillos a rojizos, de 1.8 a $2.5 \mathrm{~cm}$ de largo; brácteas y bractéolas deciduas. Flores 9 a 20 por inflorescencia; pedicelo de 4 a $6 \mathrm{~mm}$ de largo, por lo general la flor central del dicasio sésil, botón globoso, apiculado, de 2 a $3 \mathrm{~mm}$ de largo, por $\pm 2 \mathrm{~mm}$ de ancho, caliptra en forma de cúpula, membranácea, glandulosa, hasta $3.5 \mathrm{~mm}$ de ancho; cáliz cerrado en el botón; pétalos ausentes, estambres 80 a 100, de hasta $4 \mathrm{~mm}$ de largo; estilo de $\pm 5 \mathrm{~mm}$ de largo, ovario bilocular, óvulos 2 por lóculo; hipantio campanulado, de $\pm 1.5 \mathrm{~mm}$ de largo, por $\pm 1 \mathrm{~mm}$ de ancho en el ápice, glabro, glanduloso. Fruto globoso, inmaduro verde, rojo al madurar, de 5 a $9 \mathrm{~mm}$ de diámetro, glabro, coronado con el hipantio en el ápice. Semilla 1, radícula alargada, cotiledones foliáceos, contortuplicados.

Material adicional examinado. MÉXICO. Querétaro: municipio de Arroyo Seco: río Santa María, aprox. 3 a 4 km río abajo del Puente Concá, E. Carranza 1786 (ENCB, IBUG, IEB, MEXU, QMEX); río Santa María, $\pm 3 \mathrm{~km}$ al NE de las Adjuntas con el río Concá, S. Zamudio y E. Sánchez 16358 (IEB); municipio de Jalpan: La Isla, en la orilla del río Santa María, $6 \mathrm{~km}$ al N de El Carrizal, R. Fernández 4473 (ENCB, IEB); al SO de Tanchanaquito en la desembocadura del cañón de Tanquizul al río, L. López 312 (IEB). Hidalgo: municipio de Pacula: camino de descenso al río Moctezuma, vereda de Pacula-Rancho la Peña-Río Moctezuma, A. Castro-Castro 772 (IBUG). 
Calyptranthes moctezumae sobre todo ha sido colectada en el extremo nororiental de Querétaro, en donde es un elemento abundante en cañadas y laderas de roca caliza entre el bosque tropical caducifolio y el matorral submontano, con menor presencia en el bosque tropical subcaducifolio. Habita en los cañones de los ríos que forman parte de la cuenca del río Pánuco, como son el río Concá, en el municipio de Arroyo Seco y el Santa María en el de Jalpan, encontrándose también en el del río Moctezuma en el municipio de Pacula, Hidalgo. Crece en elevaciones de 250 a 600 $\mathrm{m}$. Se le ha encontrado en floración de marzo a junio, con frutos a partir de mayo. Debido a su distribución limitada y a las amenazas a que están sujetos el bosque tropical caducifolio y subcaducifolio de la zona de estudio se le considera vulnerable a la extinción.

Además de C. moctezumae, 24 especies de Calyptranthes se distribuyen en México y pueden distinguirse entre otros caracteres por el tipo de indumento que presentan. Morfológicamente esta especie está relacionada con C. schlechtendaliana O. Berg y C. schiedeana O. Berg, con las que se puede confundir pues comparten la condición glabra en ramillas e inflorescencias. La confusión entre estas entidades no solo se debe a la semejanza morfológica, sino también a su distribución, dado que las tres se encuentran en el sur de la Sierra Madre Oriental. Sin embargo, C. moctezumae presenta varias características claramente distintivas como son las hojas elípticas a subrómbicas, menor número de nervaduras por hoja, y habita en el bosque tropical caducifolio, subcaducifolio y matorral submontano. Otras diferencias se encuentran en el Cuadro 1.

El nombre de la especie hace referencia al río Moctezuma, en cuyo cañón es una planta común.

Eugenia queretaroana E. Sánchez-Chávez \& Zamudio sp. nov. Fig. 2.

Tipo: MÉXICO. Querétaro, municipio de Landa, $3 \mathrm{~km}$ al SE de San Juan Los Tubos, 17 junio 1990, H. Rubio 1595 (holotipo: IEB; isotipos: MEXU, QMEX).

Arbor vel frutex 4.5-15(-25) m altus; cortex brunneo-rubescens leviter fisuratus; ramuli compressi vel teretes hirtuli trichomatibus brevibus simplicibus cupreis aetate albescentibus persistentibus ut in petiolo, costa et inflorescentia. Petiolus teres (4.5-)5-7(-8) mm longus, folii lamina ovato-elliptica ad elliptica 4.5-8.7(-10) cm longa, 2.1-4.9 cm lata, margine undulato et revoluto. Inflorescentiae axillares, racemis 1-2 per axillam, interdum in ramorum defoliatum nodis, axis angulosus (1-)2-7.5 $\mathrm{mm}$ longus, bracteolae persistentes deltatae ad lanceolatae 0.5-1 mm longae. Flores 
Cuadro 1. Principales diferencias entre Calyptranthes schiedeana, C. schlechtendaliana y C. moctezumae.

\begin{tabular}{|c|c|c|c|}
\hline Carácter & C. schiedeana & C. schlechtendaliana & C. moctezumae \\
\hline Forma de crecimiento & árbol de 2-15 m & $\begin{array}{l}\text { arbusto o árbol de } \\
2-6 \mathrm{~m}\end{array}$ & arbusto de $1-4 \mathrm{~m}$ \\
\hline Ramillas, forma & teretes, 2-costilladas & comprimidas o teretes & $\begin{array}{l}\text { comprimidas o } \\
\text { teretes, } 2 \text {-costilladas }\end{array}$ \\
\hline Peciolo, largo & $3.5-6 \mathrm{~mm}$ & $3.5-6 \mathrm{~mm}$ & $1-5 \mathrm{~mm}$ \\
\hline Hoja, forma & ovado-elíptica & $\begin{array}{l}\text { obovada u obovado- } \\
\text { lanceolada }\end{array}$ & elíptica a subrómbica \\
\hline Hojas, largo $\times$ ancho & $3.7-8.3 \times 2.1-4.3 \mathrm{~cm}$ & $3.2-6.5 \times 1-2.4 \mathrm{~cm}$ & $2.3-5.3 \times 1-2.2 \mathrm{~cm}$ \\
\hline $\begin{array}{l}\text { Núm. de nervios } \\
\text { laterales }\end{array}$ & $25-35$ por lado & $17-24$ por lado & $10-15$ por lado \\
\hline $\begin{array}{l}\text { Ángulo de las } \\
\text { nervaduras laterales } \\
\text { con respecto de la } \\
\text { central }\end{array}$ & más de $45^{\circ}$ & menos de $45^{\circ}$ & más de $45^{\circ}$ \\
\hline Textura de la hoja & cartácea & coriácea & coriácea \\
\hline Tipo de glándulas & pelúcido-punteadas & impresas-punteadas & pelúcido-punteadas \\
\hline Inflorescencia, largo & $4.5-8 \mathrm{~cm}$ & $3-5 \mathrm{~cm}$ & $2-5 \mathrm{~cm}$ \\
\hline $\begin{array}{l}\text { Núm. de flores por } \\
\text { inflorescencia }\end{array}$ & $30-50$ & $10-15$ & $9-20$ \\
\hline Pedúnculo, largo & $2-4 \mathrm{~cm}$ & $1-2.2 \mathrm{~cm}$ & $1.8-2.5 \mathrm{~cm}$ \\
\hline Botón, largo & $1.3 \mathrm{~mm}$ & hasta $4 \mathrm{~mm}$ & $1.3 \mathrm{~mm}$ \\
\hline Hábitat & $\begin{array}{l}\text { bosque de encino, } \\
\text { bosque tropical } \\
\text { subcaducifolio, } \\
\text { bosque tropical } \\
\text { caducifolio y } \\
\text { vegetación riparia }\end{array}$ & $\begin{array}{l}\text { bosque de encino, } \\
\text { bosque tropical } \\
\text { perennifolio, } \\
\text { bosque tropical } \\
\text { subcaducifolio y } \\
\text { bosque tropical } \\
\text { caducifolio }\end{array}$ & $\begin{array}{l}\text { bosque tropical } \\
\text { caducifolio, } \\
\text { subcaducifolio y } \\
\text { matorral submontano }\end{array}$ \\
\hline
\end{tabular}

3-9 per inflorescentiam, pediceli 1-4 mm longi; calycis lobuli deltati, hypanthium infundibuliforme. Fructus ellipsoideus, sui basi turbinata stipitem falsum ad $3 \mathrm{~mm}$ longum in maturitate formanti.

Árbol o arbusto de 4 a 15(-25) m de altura; corteza pardo-rojiza, ligeramente fisurada; ramillas comprimidas o teretes, lisas, hírtulas, glabrescentes con la edad; indumento con tricomas simples cortos, cobrizos, blanquecinos con la edad, persis- 
Sánchez-Chávez y Zamudio: Tres especies nuevas de Myrtaceae

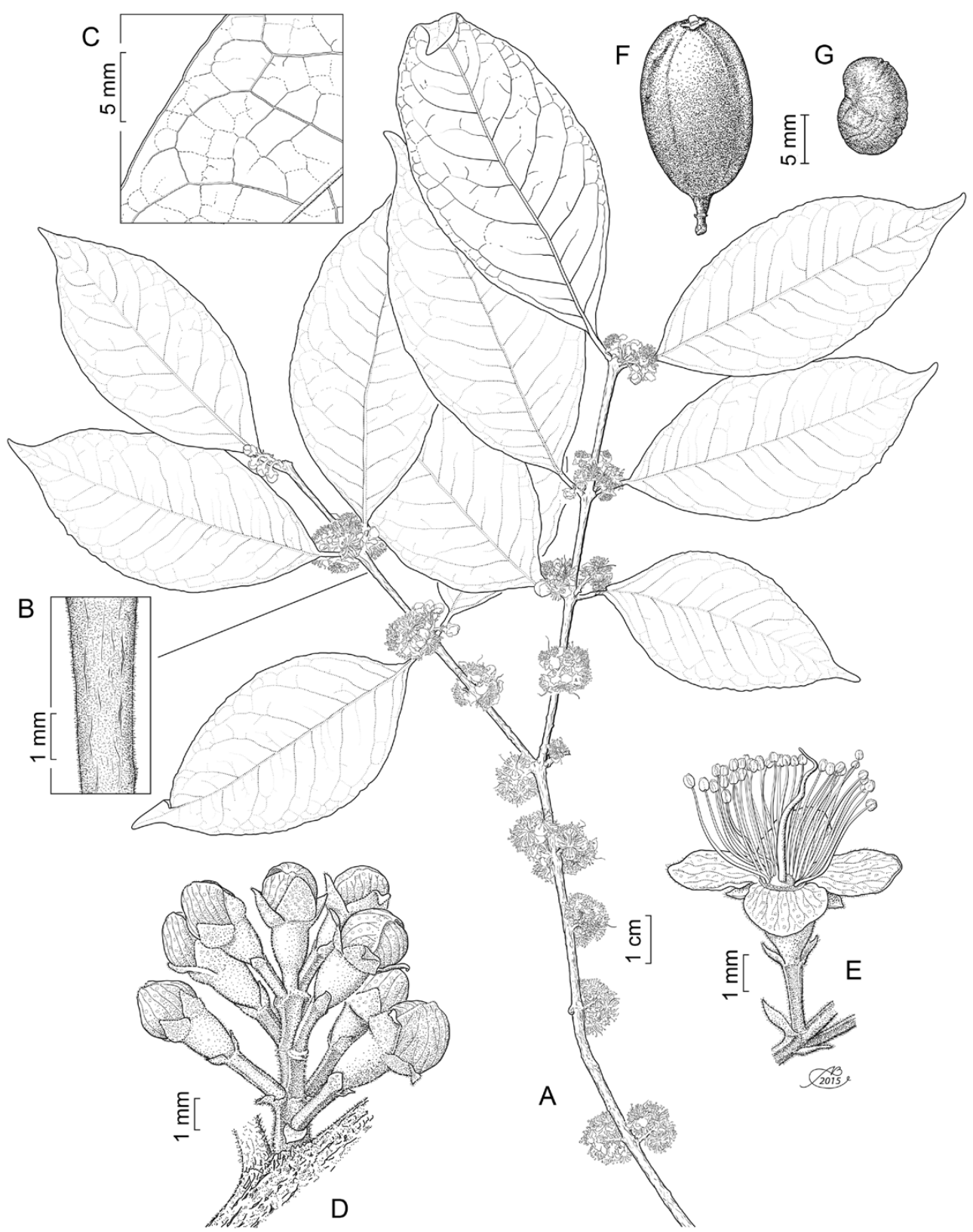

Fig. 2. Eugenia queretaroana E. Sánchez-Chávez \& Zamudio. A. rama con inflorescencias; B. acercamiento de la rama mostrando la pubescencia; C. acercamiento del envés de la hoja mostrando el margen revoluto y la venación terciaria; D. inflorescencia con flores en botón, se aprecia el eje anguloso; E. flor; F. fruto; G. semilla. Ilustración de Alfonso Barbosa. 
tiendo en las ramillas, peciolo, vena media e inflorescencia. Peciolo terete, de (4.5-)5 a 7(-8) $\mathrm{mm}$ de largo, por 0.8 a $1 \mathrm{~mm}$ de ancho; lámina foliar verde oscura, lustrosa en el haz, pálida y opaca en el envés, elíptica a ovado-elíptica, de 4.5 a $8.7(-10) \mathrm{cm}$ de largo, por $2.1 \mathrm{a} 4.9 \mathrm{~cm}$ de ancho, ápice acuminado, a veces falcado, base cuneada a obtusa, margen ondulado, revoluto, vena media cóncava en el haz, convexa en el envés, nervios laterales 5 a 10(-12) por lado, escasamente cóncavos en el haz, convexos en el envés, nervio marginal arqueado entre los laterales, distante 1.5 a 5 mm del margen, coriácea, glabra en el haz, glabrescente en el envés, con glándulas convexas en ambas superficies. Inflorescencias axilares, 1 a 2 racimos cortos por axila, a veces en los nudos de ramas defoliadas; eje de la inflorescencia anguloso, de (1-)2 a $7.5 \mathrm{~mm}$ de largo, hírtulo, con tricomas adpresos, más densos en la base; brácteas subpersistentes, ovado-lanceoladas, ápice agudo a redondeado, de $0.4 \mathrm{a} 1 \mathrm{~mm}$ de largo, ciliadas; bractéolas persistentes, deltadas a lanceoladas, de 0.5 a $1 \mathrm{~mm}$ de largo, hírtulas, cilioladas. Flores 3 a 9 por inflorescencia; pedicelos de 1 a $4 \mathrm{~mm}$ de largo, hírtulos, botón globoso, de 1.5 a $2 \mathrm{~mm}$ de largo, por $\pm 2 \mathrm{~mm}$ de ancho; lóbulos del cáliz en pares subiguales o desiguales, deltados, el par más pequeño de 0.9 a 1.1 $\mathrm{mm}$ de largo, por 1 a $1.3 \mathrm{~mm}$ de ancho, el par más grande de 1 a $1.6 \mathrm{~mm}$ de largo, por 1 a $1.3 \mathrm{~mm}$ de ancho, ciliados, glandulosos; pétalos blancos, ovado-elípticos, de 2 a $4 \mathrm{~mm}$ de largo, por 1.5 a $3 \mathrm{~mm}$ de ancho, ciliados, glandulosos, estambres \pm 50 , de 4 a 6(-8) $\mathrm{mm}$ de largo, disco glabrescente, de 1 a $2 \mathrm{~mm}$ de diámetro; estilo de 4 a $7 \mathrm{~mm}$ de largo, glabro, ovario bilocular, óvulos 4 a 10 por lóculo; hipantio infundibuliforme, de 1 a $1.5 \mathrm{~mm}$ de largo, por 0.8 a $1.3 \mathrm{~mm}$ de ancho en la parte más amplia, hírtulo. Fruto elipsoidal, rojizo cuando joven, negro al madurar, de 1 a $2 \mathrm{~cm}$ de largo, por 0.8 a $1.5 \mathrm{~cm}$ de diámetro, la base turbinada forma un falso estípite de hasta $3 \mathrm{~mm}$ de largo en los frutos maduros, pericarpio delgado, glabro, glandular, lóbulos del cáliz persistentes, bractéolas persistentes. Semilla 1, embrión homogéneo, radícula y cotiledones connados.

Material adicional examinado: MÉXICO. Querétaro: municipio de Jalpan: 2-3 km al Poniente de El Saucito, B. Servín 211 (IEB, QMEX); 1-1.5 km al E de El Saucito, E. Carranza 2217 (IEB, QMEX); 4-5 km al N de La Parada, punto Los Bancos, B. Servín 420 (IEB, MEXU, QMEX); 2-3 km al N de La Parada, B. Servín 509 (IEB, QMEX); 4-5 km al O de La Parada, punto La Peña, B. Servín 362 (IEB, MEXU); 2-3 km al poniente de San Isidro, La Parada, B. Servín 204 (IEB, MEXU, QMEX); $2 \mathrm{~km}$ al NO del Embocadero, E. González 1460 (IEB, QMEX); al N del Carrizal, camino al Naranjo, C. Guzmán 99 (IEB, QMEX); municipio de Landa: $2 \mathrm{~km}$ al NO de El Rincón, H. Rubio 806 (EBUM, IBUG, IEB, QMEX); $1.5 \mathrm{~km}$ al Poniente de Jagüey Colorado, H. Rubio 1310 (IEB, MEXU, QMEX); $2.5 \mathrm{~km}$ al SE 
de San Juan Los Tubos, H. Rubio 1920 (IEB, QMEX); $2 \mathrm{~km}$ al S de Neblinas, $H$. Rubio 252 (IEB, MEXU, QMEX); $6 \mathrm{~km}$ al $\mathrm{S}$ de La Lagunita rumbo a Tilaco, $E$. Carranza 611 (IEB); municipio de Pinal de Amoles: $9 \mathrm{~km}$ al S de Santa Águeda sobre el camino a Ahuacatlán, J. Rzedowski 46633 (IEB, MEXU); La Cuesta, 3 km al S de Escanelilla, R. Fernández 1578 (ENCB, IBUG, IEB, MEXU); 1647 (ENCB, IEB, MEXU).

Se conocen varias poblaciones de Eugenia queretaroana en el extremo nororiental del estado de Querétaro, en laderas de cerros cercanas a arroyos, en regiones con clima templado subhúmedo, en donde es un elemento abundante dentro de los bosques de pino-encino y mesófilo de montaña; se puede encontrar también en la transición entre el bosque mesófilo de montaña y el bosque tropical subcaducifolio. Crece en elevaciones de 800 a $1450 \mathrm{~m}$. Se le conoce en floración en los meses de abril a junio; los frutos jóvenes se encuentran de junio a agosto y los maduros de septiembre a noviembre. Planta localmente abundante en el extremo noreste de Querétaro; sin embargo, debido a las amenazas a las que están expuestos los bosques mesófilo de montaña y tropical subcaducifolio en la región de estudio se considera vulnerable.

El nombre propuesto hace alusión al estado de Querétaro, de donde proceden los ejemplares de esta planta.

Especie morfológicamente similar a Eugenia crenularis Lundell y E. pueblana Lundell; sin embargo, E. queretaroana se distingue por el indumento cobrizo, peciolo terete, hipantio infundibuliforme y frutos elipsoidales con un falso estípite en la base. Existe una marcada diferencia ecológica entre E. pueblana, que habita en zonas con clima cálido subhúmedo, y E. queretaroana que prospera en el clima templado subhúmedo. Asimismo hay una separación geográfica con E. crenularis, que crece en la provincia fisiográfica del Eje Neovolcánico, a diferencia de E. queretaroana que se encuentra en la Sierra Madre Oriental. Otras características distintivas se pueden apreciar en el Cuadro 2.

Eugenia huasteca E. Sánchez-Chávez \& Zamudio sp. nov. Fig. 3.

Tipo: MÉXICO. Querétaro, municipio de Jalpan, La Vuelta de la Peña cerca de Tanchanaquito, 16 julio 2014, E. Sánchez-Chávez y S. Zamudio 15 (holotipo: IEB; isotipos: ENCB, MEXU, QMEX, UAMIZ).

Frutex 2-4 m altus; cortex brunneo-rubellus ad griseus leviter fisuratus; ramuli teretes ad leviter compressi hirtuli vel pilosi, hoc indumentum persistens ut in 
Cuadro 2. Principales diferencias entre Eugenia crenularis, E. pueblana y E. queretaroana.

\begin{tabular}{|c|c|c|c|}
\hline Carácter & E. crenularis & E. pueblana & E. queretaroana \\
\hline Ramillas, forma & comprimidas & comprimidas & $\begin{array}{l}\text { comprimidas o } \\
\text { teretes }\end{array}$ \\
\hline Indumento, color & rojizo & rojizo & $\begin{array}{l}\text { cobrizo, } \\
\text { blanquecino con } \\
\text { la edad }\end{array}$ \\
\hline Peciolo, forma & acanalado & $\begin{array}{l}\text { terete o aplanado } \\
\text { adaxialmente }\end{array}$ & terete \\
\hline Margen, lámina foliar & $\begin{array}{l}\text { aparentemente } \\
\text { crenulado, revoluto }\end{array}$ & revoluto & $\begin{array}{l}\text { revoluto y } \\
\text { ondulado }\end{array}$ \\
\hline $\begin{array}{l}\text { Eje de la inflorescencia, } \\
\text { largo }\end{array}$ & $1-4.5 \mathrm{~mm}$ & $\begin{array}{l}0-2 \mathrm{~mm} \text { (a veces en } \\
\text { los nudos de ramitas } \\
\text { viejas) }\end{array}$ & (1-)2-7.5 mm \\
\hline Bractéolas, forma & ovadas & deltadas a orbiculares & $\begin{array}{l}\text { ovado-deltadas a } \\
\text { lanceoladas }\end{array}$ \\
\hline $\begin{array}{l}\text { Núm. de flores por } \\
\text { inflorescencia }\end{array}$ & $1-3$ & $2-4$ & $3-9$ \\
\hline $\begin{array}{l}\text { Pedicelos, largo, e } \\
\text { indumento }\end{array}$ & $\begin{array}{l}\text { 1-2 } \mathrm{mm} \text {, glabro o } \\
\text { pubescente }\end{array}$ & $\begin{array}{l}0.5-1.3 \mathrm{~mm} \text {, } \\
\text { pubescente }\end{array}$ & $\begin{array}{l}\text { 1-4 mm, } \\
\text { pubescente }\end{array}$ \\
\hline $\begin{array}{l}\text { Lóbulos del cáliz, } \\
\text { forma }\end{array}$ & redondos & redondos & deltados \\
\hline Botón, forma & piriforme & piriforme & globoso \\
\hline Hipantio, forma & campanulado & cortamente estipitado & infundibuliforme \\
\hline Fruto, forma & elipsoidal & globoso & $\begin{array}{l}\text { elipsoidal, con } \\
\text { un falso estípite } \\
\text { de hasta } 3 \mathrm{~mm} \text { de } \\
\text { largo en la base }\end{array}$ \\
\hline Fruto, largo $\times$ ancho & $0.9 \times 0.7-1 \mathrm{~cm}$ & $1.3 \times 1.6 \mathrm{~cm}$ & $1.5-2 \times 0.9-1.5 \mathrm{~cm}$ \\
\hline
\end{tabular}

petiolo, costa, nerviis lateralibus et inflorescentia. Folii lamina ovata ad elliptica, 3-7 cm longa, 2-4.2 cm lata, basi rotundata ad obtusa vel leviter cuneata, coriacea. Inflorescentiae axillares, racemo solitario per axillam, axis 0-3 mm longus; bracteae deciduae cum indumento ad $0.5 \mathrm{~mm}$ longo; bracteolae persistentes connatae involucrum formantes. Flores 6-8 per inflorescentiam, sessiles, hypanthio cupulato 0.5-1 $\mathrm{mm}$ longo, 1-1.5 mm lato, indumento ad $0.8 \mathrm{~mm}$ longo adpresso.

Arbusto de 2 a $4 \mathrm{~m}$ de alto, tallo de 2 a $3 \mathrm{~cm}$ de diámetro; corteza pardo-rojiza a gris, ligeramente fisurada; ramillas teretes o ligeramente comprimidas, las ter- 


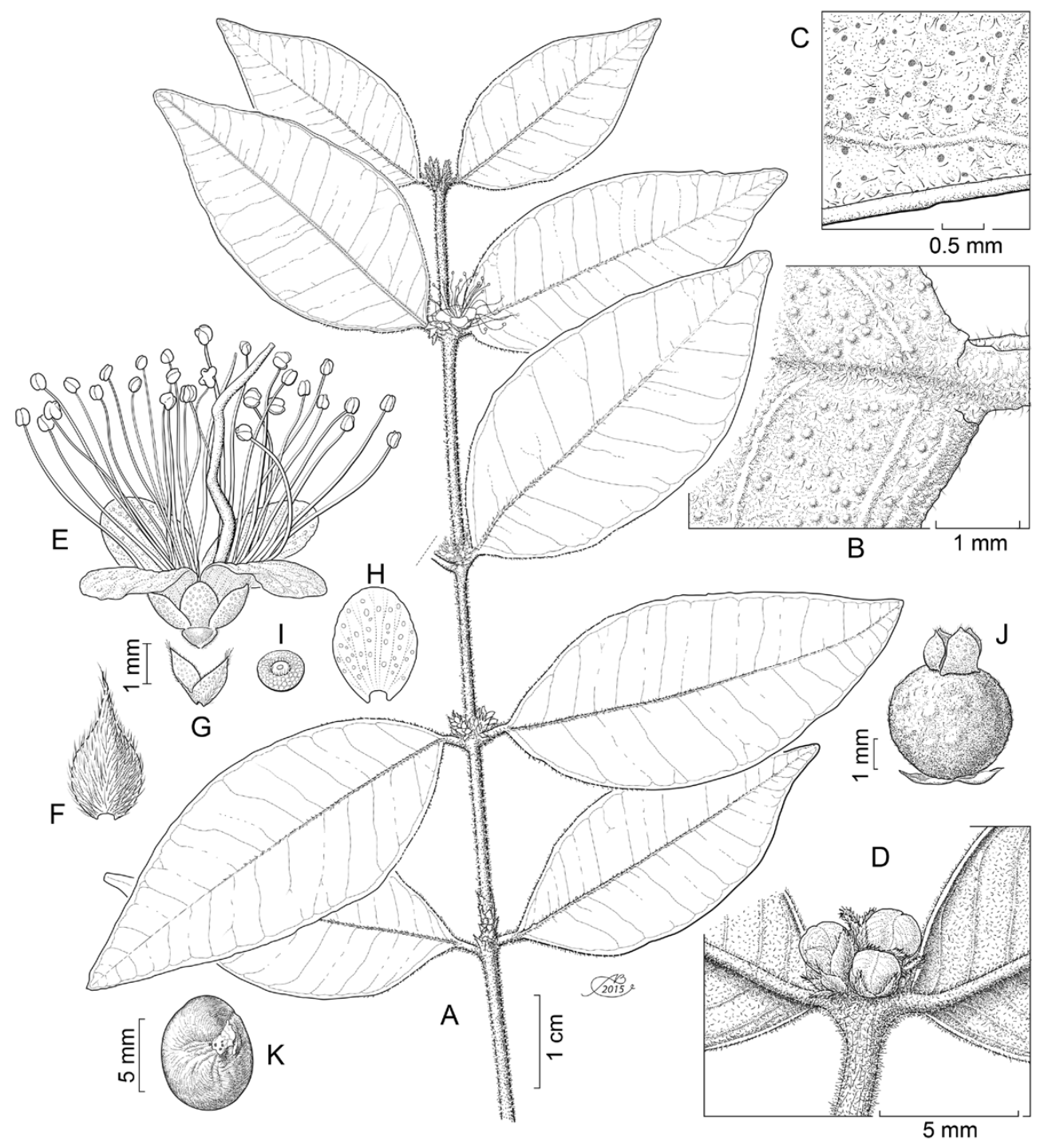

Fig. 3. Eugenia huasteca E. Sánchez-Chávez \& Zamudio. A. rama con flores; B. acercamiento del haz de la hoja mostrando las glándulas y pubescencia; C. acercamiento del envés de la hoja mostrando el margen revoluto, las glándulas y la pubescencia; D. acercamiento mostrando la flores sésiles en botón y la pubescencia; E. flor; F. bráctea; G. bractéolas unidas en la base; H. pétalo; I. disco mostrando lo puntos de inserción de los estambres; J. fruto; K. semilla. Ilustración de Alfonso Barbosa. 
minales hírtulas o pilosas, con tricomas simples, pardo-rojizos, blanquecinos con la edad, persistiendo en el peciolo, vena media, nervios laterales e inflorescencia. Peciolo terete, de 2.5 a $5 \mathrm{~mm}$ de largo, por $1 \mathrm{~mm}$ de ancho; lámina foliar de color verde oscuro en el haz, verde claro en el envés, ovada a elíptica, de 3 a $7 \mathrm{~cm}$ de largo, por 2 a $4.2 \mathrm{~cm}$ de ancho, ápice agudo, acuminado, base redondeada a obtusa o ligeramente cuneada, margen ligeramente revoluto, vena media impresa en el haz y convexa en el envés, nervios laterales 6 a 10 por lado, convexos en el haz y envés, nervio marginal levemente arqueado, distante $\pm 1 \mathrm{~mm}$ del margen, coriácea, pubescente en ambas superficies. Inflorescencias axilares, 1 racimo por axila, indumento adpreso; eje sésil o hasta $3 \mathrm{~mm}$ de largo; brácteas deciduas, pardo-rojizas, lanceoladas, de (1.3-)1.8 a $2 \mathrm{~mm}$ de largo, por 0.5 a $1 \mathrm{~mm}$ de ancho, pilosas, ciliadas, indumento de hasta $0.5 \mathrm{~mm}$ de largo, superficie interna glabra, glándulas convexas, rígidas; bractéolas persistentes, cóncavas, ovadas, ápice agudo a redondeado, cilioladas, connadas formando un involucro, de 1 a $1.5 \mathrm{~mm}$ de alto, por 1 a $1.3 \mathrm{~mm}$ de ancho, superficie interna glabra, la externa pubescente, la pubescencia más densa en la base, con glándulas translucidas conspicuas. Flores 6 a 8 por inflorescencia, sésiles, botón globoso, de $3 \mathrm{~mm}$ de alto, por $2.5 \mathrm{~mm}$ de ancho, lóbulos del cáliz en pares subiguales o desiguales, dentados, el par más pequeño de 0.7 a $1 \mathrm{~mm}$ de largo, por $1 \mathrm{a} 1.5 \mathrm{~mm}$ de ancho, el par más grande de $1 \mathrm{a} 1.5 \mathrm{~mm}$ de alto, por $1.5 \mathrm{a} 2 \mathrm{~mm}$ de ancho, glándulas conspicuas translucidas, ciliados, superficie interna glabra, externa glabrescente; pétalos blancos, glabros excepto por algunos tricomas en el ápice, glándulas inconspicuas planas, de 2 a $3.5 \mathrm{~mm}$ de largo, por 1.3 a $2.5 \mathrm{~mm}$ de ancho, estambres de 30 a 40, $\pm 5 \mathrm{~mm}$ de largo, disco redondo, de 1.3 a $2 \mathrm{~mm}$ de ancho, híspido en su derredor; estilo glabro, de $6 \mathrm{~mm}$ de largo, ovario bilocular, óvulos 10 por lóculo; hipantio en forma de copa, de 0.5 a $1 \mathrm{~mm}$ de alto, por 1 a 1.5 $\mathrm{mm}$ de ancho, densamente pubescente, indumento hasta $0.8 \mathrm{~mm}$ de largo, adpreso, glándulas inconspicuas. Fruto globoso, verde a rojo cuando inmaduro, negro al madurar, de 7 a $8 \mathrm{~mm}$ de diámetro; pericarpio de paredes delgadas, glanduloso, glabrescente, lóbulos del cáliz persistentes.

Material adicional examinado: MÉXICO. Querétaro: municipio de Jalpan, al oriente de Tanchanaquito, paraje La Vuelta de la Peña, L. López 414 (IEB); La Vuelta de la Peña, camino a Tanchanaquito, S. Zamudio 16500 (IEB); La Vuelta de la Peña, Tanchanaquito, E. Sánchez y S. Zamudio 16 (ENCB, IEB, MEXU, QMEX); La Vuelta de la Peña, Tanchanaquito, E. Sánchez y S. Zamudio 17 (ENCB, IBUG, IEB, MEXU, QMEX, UAMIZ).

Eugenia huasteca se conoce de una sola localidad cercana a Tanchanaquito, al norte del municipio de Jalpan, Querétaro, en las proximidades del límite con San 
Luis Potosí. Habita en zonas de clima cálido subhúmedo con bosque tropical subcaducifolio de Brosimum alicastrum. Crece en elevaciones de 200 a $400 \mathrm{~m}$. Se ha encontrado en floración de mayo a julio y en fructificación de julio a septiembre. Debido a su distribución tan restringida y a lo reducido de su población se considera vulnerable a la extinción.

La especie nueva se puede relacionar morfológicamente con Eugenia capuli (Schltdl. \& Cham.) Hook \& Arn.; sin embargo, E. huasteca es densamente pubescente, con indumento simple, pardo-rojizo a blanquecino y flores sésiles, características claramente distintivas de cualquier otra especie de este género; además, existen discrepancias fenológicas ya que E. huasteca se encuentra en floración durante los meses de mayo a julio, y E. capuli, en esa localidad, durante los meses de noviembre y diciembre. Otras diferencias se encuentran en el Cuadro 3.

Cuadro 3. Principales diferencias entre Eugenia capuli y E. huasteca.

\begin{tabular}{|c|c|c|}
\hline Carácter & E. capuli & E. huasteca \\
\hline Indumento, forma y color & $\begin{array}{l}\text { hispídulo con pelos simples, } \\
\text { erectos, pálidos }\end{array}$ & $\begin{array}{l}\text { hírtulo o piloso, pelos } \\
\text { simples, pardo rojizos a } \\
\text { blanquecinos con la edad }\end{array}$ \\
\hline Núm. de nervios laterales & $4-8$ & $6-10$ \\
\hline $\begin{array}{l}\text { Eje de la inflorescencia, } \\
\text { largo }\end{array}$ & $1-10 \mathrm{~mm}$ & $0-3 \mathrm{~mm}$ \\
\hline Brácteas, largo & ca. $0.3 \mathrm{~mm}$ & $(1.3-) 1.8-2 \mathrm{~mm}$ \\
\hline Pedicelos, largo & $2-7 \mathrm{~mm}$ & ausentes \\
\hline
\end{tabular}

\section{AGRADECIMIENTOS}

Los autores agradecen al Dr. Jerzy Rzedowski por la revisión del artículo y las diagnosis latinas, a Alfonso Barbosa por las excelentes ilustraciones. Se agradece también a los curadores de los herbarios EBUM, ENCB, IBUG, IEB, MEXU y QMEX por las facilidades otorgadas para la consulta y préstamo de ejemplares. Al Instituto de Ecología, A. C. (cuenta 20006), a la Comisión Nacional para el Conocimiento y Uso de la Biodiversidad (CONABIO) y al Consejo Nacional de Ciencia y Tecnología (CONACyT) por el financiamiento para esta investigación. 


\section{LITERATURA CITADA}

Landrum, L. R., F. Barrie, L. Kawasaki y K. Holst. 2009. Myrtaceae. In: Davidse, G., M. Sousa, S. Knapp y F. Chiang. (eds.). Cucurbitaceae a Polemoniaceae. Flora Mesoamericana 4: 66-150.

Mazine, F. F., V. Castro Souza, M. Sobral, F. Forest y E. Lucas. 2014. A preliminary phylogenetic analysis of Eugenia (Myrtaceae: Myrteae), with a focus on Neotropical species. Kew Bulletin 69: 94-97

McVaugh, R. 1963. Tropical American Myrtaceae, II. Fieldiana, Botany 29(8): 393-532.

Recibido en octubre de 2015.

Aceptado en febrero de 2016. 